\title{
FITOTECNIA
}

\section{SELETIVIDADE DA SOJA TRANSGÊNICA TOLERANTE AO GLYPHOSATE E EFICÁCIA DE CONTROLE DE COMMELINA BENGHALENSIS COM HERBICIDAS APLICADOS ISOLADOS E EM MISTURAS $\left({ }^{1}\right)$}

\author{
NÚBIA MARIA CORREIA $\left(\left(^{*}\right)\right.$; JULIO CEZAR DURIGAN $\left({ }^{2}\right)$; GILSON JOSÉ LEITE $\left({ }^{3}\right)$
}

\author{
RESUMO
}

O objetivo do trabalho foi avaliar os possíveis efeitos fitotóxicos de herbicidas aplicados em pósemergência, isolados e em misturas, na soja transgênica (cv. M-SOY 8008 RR) tolerante ao glyphosate e no controle de plântulas de Commelina benghalensis. Os experimentos foram desenvolvidos no período de janeiro a maio de 2006, em vasos, mantidos em condições de ambiente não controlado, no Departamento de Fitossanidade, UNESP, Campus de Jaboticabal (SP). Os tratamentos avaliados foram: glyphosate $(1,20$ $\mathrm{kg} \mathrm{ha}^{-1} \mathrm{e}$.a.), chlorimuron-ethyl $\left(0,02 \mathrm{~kg} \mathrm{ha}^{-1}\right)$, lactofen $\left(0,18 \mathrm{~kg} \mathrm{ha}^{-1}\right)$, fomesafen $\left(0,25 \mathrm{~kg} \mathrm{ha}^{-1}\right)$, flumioxazin $\left(0,025 \mathrm{~kg} \mathrm{ha}^{-1}\right)$, imazethapyr $\left(0,10 \mathrm{~kg} \mathrm{ha}^{-1}\right)$, chlorimuron-ethyl $\left(0,01 \mathrm{~kg} \mathrm{ha}^{-1}\right)$ mais lactofen $\left(0,096 \mathrm{~kg} \mathrm{ha}^{-1}\right)$, chlorimuron-ethyl $\left(0,01 \mathrm{~kg} \mathrm{ha}^{-1}\right)$ mais fomesafen $\left(0,125 \mathrm{~kg} \mathrm{ha}^{-1}\right)$, lactofen $\left(0,096 \mathrm{~kg} \mathrm{ha}^{-1}\right)$ mais fomesafen $\left(0,125 \mathrm{~kg} \mathrm{ha}^{-1}\right)$, as misturas de glyphosate $\left(0,60 \mathrm{kgha}^{-1}\right)$ com chlorimuron-ethyl $\left(0,01 \mathrm{~kg} \mathrm{ha}^{-1}\right)$, lactofen $\left(0,096 \mathrm{~kg} \mathrm{ha}^{-1}\right)$, fomesafen $\left(0,125 \mathrm{~kg} \mathrm{ha}^{-1}\right)$, flumioxazin $\left(0,0125 \mathrm{~kg} \mathrm{ha}^{-1}\right)$, imazethapyr $\left(0,05 \mathrm{~kg} \mathrm{ha}^{-1}\right)$ e uma testemunha sem aplicação de herbicida. No experimento com $C$. benghalensis, testou-se também a aplicação seqüencial de glyphosate $\left(0,96\right.$ mais $0,72 \mathrm{~kg} \mathrm{ha}^{-1}$ e.a.). A associação de ghyphosate a herbicidas utilizados em soja convencional ocasionou danos visuais às plantas de soja. Em alguns casos, como nas misturas de glyphosate com lactofen e glyphosate com flumioxazin, os sintomas foram severos, com necroses e pontos cloróticos nas folhas. Mesmo assim, essa fitointoxicação não influenciou no desenvolvimento vegetativo e reprodutivo da soja. A mistura de glyphosate a outros herbicidas, assim como a sua aplicação seqüencial, não foram eficazes no controle de C. benghalensis, nas doses testadas e quando as plantas estavam no estádio de 4 a 6 folhas totalmente expandidas.

Palavras-chave: controle químico, Roundup Ready, sintomas de fitointoxicação, soja transgênica, trapoeraba.

$\left({ }^{1}\right)$ Recebido para publicação em 7 de novembro de 2006 e aceito em 14 de abril de 2008.

$\left({ }^{2}\right)$ Departamento de Fitossanidade, Universidade Estadual Paulista (UNESP). Via de Acesso Prof. Paulo Donato Castellane, s/n. ${ }^{\circ}, 14884-900$ Jaboticabal (SP). Brasil. E-mail: correianm@fcav.unesp.br $\left(^{*}\right)$ Autora para correspondência).

$\left({ }^{3}\right)$ Técnico Agrícola, Departamento de Fitossanidade, FCAV-UNESP, Jaboticabal (SP). 


\title{
ABSTRACT \\ SELECTIVITY OF GLYPHOSATE-TOLERANT SOYBEAN AND EFFICIENCY OF Commelina benghalensis CONTROL USING ISOLATED AND MIXED HERBICIDES
}

\begin{abstract}
The objective of this study was to evaluate phytotoxicity effects of herbicides applied during postemergence, either alone or in mixture with other chemicals in transgenic glyphosate-tolerant soybean (cv. M-SOY 8008 RR) and in Commelina benghalensis. Two experiments were carried out from January to May 2006, in pots, under uncontrolled conditions, at Plant Protection Department of UNESP, Jaboticabal (SP), Brazil. The following herbicides were used: glyphosate $\left(1,20 \mathrm{~kg}\right.$ a.e. ha $\left.{ }^{-1}\right)$, chlorimuron-ethyl $(0,02$ $\left.\mathrm{kg} \mathrm{ha}{ }^{-1}\right)$, lactofen $\left(0,018 \mathrm{~kg} \mathrm{ha}^{-1}\right)$, fomesafen $\left(0,25 \mathrm{~kg} \mathrm{ha}^{-1}\right)$, flumioxazin $\left(0,025 \mathrm{~kg} \mathrm{ha}^{-1}\right)$, imazethapyr $(0,10$ $\left.\mathrm{kg} \mathrm{ha}^{-1}\right)$, chlorimuron-ethyl $\left(0,01 \mathrm{~kg} \mathrm{ha}^{-1}\right)$ plus lactofen $\left(0,096 \mathrm{~kg} \mathrm{ha}^{-1}\right)$, chlorimuron-ethyl $\left(0,01 \mathrm{~kg} \mathrm{ha}^{-1}\right)$ plus fomesafen $\left(0,125 \mathrm{~kg} \mathrm{ha}^{-1}\right)$ and lactofen $\left(0,096 \mathrm{~kg} \mathrm{ha}^{-1}\right)$ plus fomesafen $\left(0,125 \mathrm{~kg} \mathrm{ha}^{-1}\right)$. It was also used the glyphosate $\left(0,60 \mathrm{~kg}\right.$ a.e. $\left.\mathrm{ha}^{-1}\right)$ in mixture with chlorimuron-ethyl $\left(0,01 \mathrm{~kg} \mathrm{ha}^{-1}\right)$, lactofen $(0,096 \mathrm{~kg}$ ha$\left.{ }^{1}\right)$, fomesafen $\left(0,125 \mathrm{~kg} \mathrm{ha}^{-1}\right)$, flumioxazin $\left(0,0125 \mathrm{~kg} \mathrm{ha}^{-1}\right)$ and imazethapyr $\left(0,05 \mathrm{~kg} \mathrm{ha}^{-1}\right)$ and a no treated control. Also the sequential application of glyphosate $\left(0,96\right.$ plus $0,72 \mathrm{~kg}$ a.e. ha $\left.{ }^{-1}\right)$ was used in the experiment with $C$. benghalensis. The association of glyphosate and conventional soybean herbicides caused soybean injury. In some cases, such as in the glyphosate-lactofen and glyphosate-flumioxazin mixtures, the symptoms were severe, with necrosis and clorosis. Although there were phytotoxic symptoms in the plant, the herbicide did not affect vegetative and reproductive soybean development. The mixture of glyphosate to other herbicides and its sequential application did not control C. benghalensis, when sprayed in the tested doses and in plants with 4 to 6 expanded leaves.
\end{abstract}

Key words: chemical control, intoxication symptoms, Roundup Ready, spiderwot, transgenic soybean.

\section{INTRODUÇÃO}

Desde a liberação do cultivo de soja geneticamente modificada no Brasil, uma série de questionamentos foi feita, como a seletividade de herbicidas utilizados em soja convencional e de misturas contendo esses produtos e o glyphosate a cultivares transgênicas, além do controle de plantas daninhas tolerantes ao glyphosate nessa nova tecnologia.

O glyphosate possui amplo espectro de controle de plantas daninhas, porém, o uso exclusivo desse herbicida em áreas de soja transgênica pode ter limitações. Algumas espécies podem requerer doses mais elevadas, aplicação seqüencial, ou ainda, a adição de outro herbicida para controle adequado (Ateh e Harvey, 1999).

Algumas plantas daninhas possuem tolerância natural e são controladas apenas parcialmente pelo glyphosate. Entre elas pode-se citar Commelina benghalensis (LACERDA e Victoria FilHo, 2004; Monquero et al., 2005a; Monquero et al., 2005b), Richardia brasiliensis (MONQUERO et al., 2005a), espécies do gênero Ipomoea (NoRRIs et al., 2001), Sesbania exaltata e Sida spinosa (LANIE et al., 1994), justificando a associação de glyphosate com outros herbicidas. Entretanto, no Brasil, as misturas em tanque são proibidas por lei e não possuem respaldo técnico, embora seja prática rotineira.

O estádio de desenvolvimento de plantas de I. hederacea e S. exaltata influenciou no controle das espécies pelo glyphosate tanto quando aplicado isolado como em associação com chlorimuron (VIDRINE et al., 2002). Nas plantas mais desenvolvidas constataram menor sensibilidade aos herbicidas, requerendo, principalmente de glyphosate, doses mais elevadas para obter controle satisfatório. GREY e RAYMER (2002) relataram que a adição de fomesafen à calda de glyphosate proporcionou maior percentagem de controle de $I$. coccinea. Porém, não agregou vantagens no controle de Senna obtusifolia, quando comparada à aplicação isolada de glyphosate.

Nas estratégias visando ao controle químico de plantas daninhas em soja tolerante ao glyphosate, deve-se considerar as espécies de plantas daninhas presentes na área, o estádio de seu desenvolvimento, a seletividade de herbicidas utilizados em soja convencional à soja transgênica e as misturas desses produtos com o glyphosate.

BENNETT et al. (1998), avaliando os efeitos de lactofen aplicado em pós-emergência, observaram aumento na intoxicação das plantas transgênicas, em relação à soja convencional. No mesmo trabalho, verificou-se que a mistura de chlorimuron com metribuzin e trifluralin, aplicados em pré-plantio com incorporação, não ocasionou nenhum efeito adverso às plantas de soja.

A adição de thifensulfuron à calda com glyphosate aumentou as injúrias nas plantas de soja, mas, apenas no primeiro ano do experimento. No ano seguinte, misturas de chlorimuron, imazethapyr ou thifensulfuron com glyphosate não ocasionaram danos às plantas, em comparação à testemunha sem aplicação (Lich et al., 1997). 
O uso da mistura de pendimethalin com chlorimuron e metribuzin, aplicados em pré-plantio com incorporação, em combinação com glyphosate, aplicado aos 14 e 42 dias após a emergência da soja, resultou em maior fitotoxicidade às plantas, com nota média de $11 \%$, aos 24 dias após a segunda aplicação de glyphosate, em relação aos outros tratamentos (Webster et al., 1999). No entanto, no fim do ciclo, as plantas apresentaram produtividade similar àquelas de outros programas de manejo.

Com base nos trabalhos mencionados anteriormente, houve variabilidade de resposta quando herbicidas utilizados em soja convencional foram aplicados em soja transgênica.

Os objetivos deste trabalho foram avaliar possíveis efeitos fitotóxicos de herbicidas aplicados em pós-emergência, isolados e em misturas, em soja transgênica tolerante ao glyphosate e sua eficácia no controle de plântulas de C. benghalensis.

\section{MATERIAL E MÉTODOS}

Os experimentos foram desenvolvidos no período de janeiro a maio de 2006, em vasos, mantidos em condições de ambiente não controlado, no Departamento de Fitossanidade, UNESP, Campus de Jaboticabal (SP).

Cada unidade experimental foi constituída por um vaso plástico com $21,50 \mathrm{~cm}$ de diâmetro e capacidade para quatro litros de solo. Como substrato, utilizou-se a mistura de terra, areia e esterco, respectivamente, na proporção $3: 1: 1$. Os resultados da análise química e física do substrato foram: $\mathrm{pH}$ (em $\mathrm{CaCl}_{2}$ ) 5,2; P (Resina) $39 \mathrm{mg} \mathrm{dm}^{-3} ; \mathrm{K}, \mathrm{Ca}, \mathrm{Mg} \mathrm{e} \mathrm{H}+\mathrm{Al}$ 6,$0 ; 18,0 ; 11,0$ e $28,0 \mathrm{mmol}_{\mathrm{c}} \mathrm{dm}^{-3}$, respectivamente; saturação por bases $56 \%$; matéria orgânica $22 \mathrm{~g} \mathrm{dm}^{-}$ 3. argila, limo, areia fina e areia grossa 310, 40, 320 e $330 \mathrm{~g} \mathrm{~kg}^{-1}$ respectivamente, sendo classificado como textura média. O Latossolo Vermelho-Escuro distroférrico, textura argilosa, foi coletado na camada de $0-20 \mathrm{~cm}$ do perfil do solo e peneirado em malha de $5 \mathrm{~mm}$.

A cultivar de soja utilizada foi a M-SOY 8008 RR. Foram semeadas 10 sementes por vaso, distribuídas homogeneamente, até $5 \mathrm{~cm}$ de profundidade. Posteriormente, fez-se desbaste, mantendo quatro plantas por vaso. Para C. benghalensis, foram coletadas as plântulas, provenientes de sementes, no campo e transplantadas diretamente para os vasos. Foram mantidas três plântulas por vaso. Após 15 dias do plantio, com o total restabelecimento das mudas, fez-se a aplicação dos herbicidas. No momento da pulverização, as plantas de soja estavam no estádio $\mathrm{V}_{3}$ e as de $C$. benghalensis de quatro a seis folhas totalmente expandidas.

O delineamento experimental utilizado foi o inteiramente casualizado, com quatro repetições. Os herbicidas avaliados foram: glyphosate $(1,20 \mathrm{~kg}$ ha ${ }^{1}$ e.a.), chlorimuron-ethyl $\left(0,02 \mathrm{~kg} \mathrm{ha}^{-1}\right)$, lactofen $\left(0,18 \mathrm{~kg} \mathrm{ha}^{-1}\right)$, fomesafen $\left(0,25 \mathrm{~kg} \mathrm{ha}^{-1}\right)$, flumioxazin $\left(0,025 \mathrm{~kg} \mathrm{ha}^{-1}\right)$, imazethapyr $\left(0,10 \mathrm{~kg} \mathrm{ha}^{-1}\right)$, chlorimuron-ethyl $\left(0,01 \mathrm{~kg} \mathrm{ha}^{-1}\right)$ mais lactofen $(0,096$ $\left.\mathrm{kg} \mathrm{ha}^{-1}\right)$, chlorimuron-ethyl $\left(0,01 \mathrm{~kg} \mathrm{ha}^{-1}\right)$ mais fomesafen $\left(0,125 \mathrm{~kg} \mathrm{ha}^{-1}\right)$, lactofen $\left(0,096 \mathrm{~kg} \mathrm{ha}^{-1}\right)$ mais fomesafen $\left(0,125 \mathrm{~kg} \mathrm{ha}^{-1}\right)$ e as misturas de glyphosate $\left(0,60 \mathrm{~kg} \mathrm{ha}^{-1}\right)$ com chlorimuron-ethyl $\left(0,01 \mathrm{~kg} \mathrm{ha}^{-1}\right)$, lactofen $\left(0,096 \mathrm{~kg} \mathrm{ha}^{-1}\right)$, fomesafen $\left(0,125 \mathrm{kgha}^{-1}\right)$, flumioxazin $\left(0,0125 \mathrm{kgha}^{-1}\right)$, imazethapyr $\left(0,05 \mathrm{~kg} \mathrm{ha}^{-1}\right)$. Além disso, manteve-se uma testemunha sem aplicação de herbicida. No experimento com $C$. benghalensis, testou-se também a aplicação seqüencial de glyphosate, sendo a segunda aplicação realizada 15 dias após a primeira. A descrição dos tratamentos avaliados nos experimentos é apresentada na tabela 1.

Os herbicidas foram aplicados com pulverizador costal, à pressão constante (mantida pelo $\mathrm{CO}_{2}$ comprimido) de $1,9 \mathrm{kgf} \mathrm{cm}^{-2}$, munido de barra com um bico de jato plano ("leque") de deposição uniforme 80.02 , mantido a $20 \mathrm{~cm}$ de distância do alvo, com consumo de calda equivalente a $200 \mathrm{~L} \mathrm{ha}^{-1}$. No momento da aplicação, as condições do ambiente eram: $28,1{ }^{\circ} \mathrm{C}$ de temperatura do ar, $75 \%$ de umidade relativa do ar, ventos leves com velocidade de $1,5 \mathrm{~km}$ $\mathrm{h}^{-1}$ e nebulosidade variável em torno de $25 \%$.

As plantas de soja receberam adubação de cobertura com P e K, aos 21 e 45 dias após a aplicação (DAA), e as de C. benghalensis com N, P e K, aos 21 DAA. Adicionaram-se, por vaso, $300 \mathrm{mg}$ de $\mathrm{N}$ (uréia), $600 \mathrm{mg}$ de $\mathrm{P}$ (superfosfato triplo) e $450 \mathrm{mg}$ de $\mathrm{K}$ (cloreto de potássio).

Aos 3, 7 e 14 DAA foram realizadas avaliações visuais de intoxicação nas plantas de soja, atribuindose nota em porcentagem, onde zero corresponde à ausência visual de sintomas e 100, à morte da planta. Determinou-se, aos 14 e 28 DAA, a altura das plantas, considerando-se a distância entre o colo e a extremidade da haste principal. Aos 28 DAA, quantificou-se a matéria seca da parte aérea de duas das quatro plantas de soja mantidas nos vasos. No fim do ciclo (108 DAA) das outras duas plantas, foram avaliados a altura, o número de vagens e de grãos em cada vagem, a massa de cem grãos e a produtividade de grãos por planta. 
Tabela 1. Relação dos tratamentos avaliados nos experimentos e as respectivas doses dos herbicidas (ingrediente ativo e produto comercial). UNESP, Campus de Jaboticabal (SP), 2005/2006

\begin{tabular}{llcc}
\hline & Ingredientes ativos & \multicolumn{2}{c}{ Doses } \\
\cline { 2 - 3 } Produtos comerciais & & L ou kg ha ${ }^{-1}$ & i.a. ou e.a. \\
Roundup Ready & Glyphosate & 2,50 & 1,20 \\
Roundup Ready $\left.{ }^{-1}\right)$ & Glyphosate & $2,00+1,50$ & $0,96+0,72$ \\
Classic $(2)$ & Chlorimuron-ethyl & 0,08 & 0,02 \\
Cobra & Lactofen & 0,75 & 0,18 \\
Flex (3) & Fomesafen & 1,00 & 0,25 \\
Flumizin & Flumioxazin & 0,05 & 0,025 \\
Pivot & Imazethapyr & 1,00 & 0,10 \\
Classic+Cobra & Chlorimuron-ethyl+lactofen & $0,04+0,40$ & $0,01+0,096$ \\
Classic+Flex & Chlorimuron-ethyl+fomesafen & $0,04+0,50$ & $0,01+0,125$ \\
Flex+Cobra & Fomesafen+lactofen & $0,50+0,40$ & $0,125+0,096$ \\
Roundup Ready+Classic & Glyphosate+chlorimuron-ethyl & $1,25+0,04$ & $0,60+0,01$ \\
Roundup Ready+Cobra & Glyphosate+lactofen & $1,25+0,40$ & $0,60+0,096$ \\
Roundup Ready+Flex & Glyphosate+fomesafen & $1,25+0,50$ & $0,60+0,125$ \\
Roundup Ready+Flumizin & Glyphosate+flumioxazin & $1,25+0,025$ & $0,60+0,0125$ \\
Roundup Ready+ Pivot & Glyphosate+imazethapyr & $1,25+0,50$ & $0,60+0,05$ \\
Testemunha (sem herbicida) & & & \\
\hline
\end{tabular}

(1) Aplicação seqüencial (somente no experimento de Commelina benghalensis).

(2) Adicionado de Assist 0,05\%.

() Adicionado de Energic 0,2\%.

Para C. benghalensis, foi realizada a avaliação visual da porcentagem de controle, aos 3, 7, 14, 21 e 28 DAA. Aos 56 DAA, as partes aéreas das plantas foram coletadas e secas em estufa com circulação forçada de ar, mantida a $50^{\circ} \mathrm{C}$, até massa constante.

Os resultados foram submetidos à análise de variância empregando-se o teste F. As médias dos efeitos dos herbicidas, quando significativos, foram comparadas pelo teste de Tukey a 5\% de probabilidade. Os dados de porcentagem de controle e de fitointoxicação foram transformados em $(x+1)^{1 / 2}$.

\section{RESULTADOS E DISCUSSÃO}

Para as plantas de soja, as misturas de glyphosate com os herbicidas chlorimuron-ethyl e fomesafen proporcionou leves sintomas de intoxicação, com recuperação nos primeiros dias (Tabela 2). O mesmo foi observado para as aplicações isoladas de chlorimuron-ethyl, fomesafen e imazethapyr e a mistura de chlorimuron-ethyl mais fomesafen. Contudo, a associação de ghyphosate aos herbicidas lactofen e flumioxazin, causou severa intoxicação às plantas com necrose e pontos cloróticos nas folhas. Porém, as folhas novas emitidas após a aplicação dos herbicidas mostraramse sem sintomas. Os efeitos fitotóxicos de ghyphosate mais flumioxazin foram mais evidentes que os observados com a aplicação isolada dos herbicidas. Esse fato indica que houve efeito sinérgico da mistura, ou seja, mesmo utilizando-se doses menores, a toxicidade foi superior à soma das toxicidades proporcionadas pelos produtos quando aplicados de forma isolada (VARGAS et al., 2006). O glyphosate isolado ocasionou menor injúria às plantas de soja, com nota média de $2,5 \%$.

A altura média das plantas (independentemente da época de avaliação), o acúmulo de massa seca da parte área, o número de grãos por vagem e a produtividade de grãos por planta, não foram afetados pelos herbicidas aplicados isolados ou em misturas (Tabela 3). Comprovou-se que as plantas, mesmo aquelas com maior intoxicação aos 14 DAA, conseguiram se recuperar e não apresentaram diferença no seu desenvolvimento vegetativo e na produção de grãos. 
Tabela 2. Intoxicação nas plantas de soja (cv. M-SOY 8008 RR) tolerante ao herbicida glyphosate, aos 3 , 7 e 14 dias após aplicação (DAA) de diferentes herbicidas isolados ou em mistura UNESP, Campus de Jaboticabal (SP), 2005/ 2006

\begin{tabular}{|c|c|c|c|}
\hline \multirow[t]{2}{*}{ Herbicidas } & \multicolumn{3}{|c|}{ Fitointoxicação $\left({ }^{1}\right)$} \\
\hline & 3 DAA & 7 DAA & 14 DAA \\
\hline & \multicolumn{3}{|c|}{$\%$} \\
\hline Glyphosate & $2,5 \mathrm{ab}\left({ }^{2}\right)$ & $2,5 \mathrm{ab}$ & $0,0 \mathrm{a}$ \\
\hline Chlorimuron-ethyl & $12,5 \mathrm{bcd}$ & $1,2 \mathrm{ab}$ & $0,0 \mathrm{a}$ \\
\hline Lactofen & 38,8 ef & 35,0 def & $27,5 \mathrm{bcd}$ \\
\hline Fomesafen & 20,0 cde & $11,2 \mathrm{abcd}$ & $0,0 \mathrm{a}$ \\
\hline Flumioxazin & 41,2 ef & 43,8 efg & $20,0 \mathrm{bc}$ \\
\hline Imazethapyr & $17,5 \mathrm{~cd}$ & $6,2 \mathrm{abc}$ & $2,5 \mathrm{a}$ \\
\hline Chlorimuron-ethyl+lactofen & 28,8 de & 25,0 cdef & $11,2 \mathrm{abc}$ \\
\hline Chlorimuron-ethyl+fomesafen & $7,5 \mathrm{abc}$ & 0,0 a & $0,0 \mathrm{a}$ \\
\hline Fomesafen+lactofen & 30,0 de & 16,2 bcde & $12,5 \mathrm{abc}$ \\
\hline Glyphosate+chlorimuron-ethyl & $15,0 \mathrm{~cd}$ & $2,5 \mathrm{ab}$ & $0,0 \mathrm{a}$ \\
\hline Glyphosate+lactofen & $62,5 \mathrm{fg}$ & $56,2 \mathrm{fg}$ & $30,0 \mathrm{~cd}$ \\
\hline Glyphosate+fomesafen & 25,0 de & $6,2 \mathrm{abc}$ & $0,0 \mathrm{a}$ \\
\hline Glyphosate+flumioxazin & $90,0 \mathrm{~g}$ & $85,0 \mathrm{~g}$ & $56,2 \mathrm{~d}$ \\
\hline Glyphosate+imazethapyr & 22,5 cde & $16,2 \mathrm{abcd}$ & $8,8 \mathrm{ab}$ \\
\hline Testemunha (sem herbicida) & $0,0 \mathrm{a}$ & $0,0 \mathrm{a}$ & $0,0 \mathrm{a}$ \\
\hline C.V.(\%) & 16,1 & 29,4 & 35,3 \\
\hline
\end{tabular}

( ) Os dados originais foram transformados em $(x+1)^{1 / 2}$ para realização da análise estatística. As médias apresentadas na tabela são oriundas dos dados originais.

$\left(^{2}\right)$ Médias seguidas pela mesma letra na coluna não diferem significativamente entre si pelo teste de Tukey a $5 \%$ de probabilidade.

A mistura de glyphosate com flumioxazin proporcionou o menor número de vagens por planta, mas esta diferiu apenas de chlorimuron-ethyl mais fomesafen. Como conseqüência, a associação de chlorimuron com fomesafen ocasionou a formação de grãos com menor massa, ou seja, com menor tamanho, porém, não diferiram das plantas não tratadas (Tabela 3).

VIDRINE et al. (2002) verificaram que o glyphosate aplicado sozinho não causou injúrias às plantas de soja, mas, a sua associação ao chlorimuron-ethyl (11 $\left.\mathrm{g} \mathrm{ha}^{-1}\right)$ resultou em até $6 \%$ de fitointoxicação, quando aplicados nos estádios $V_{3} \mathrm{e}$ $\mathrm{V}_{4}$, e, de $5 \%$ a $24 \%$, no estádio $\mathrm{V}_{2}$. Portanto, o estádio de desenvolvimento da soja, no momento da aplicação, pode influenciar na seletividade da associação dos herbicidas.

GREY e RAYMER (2002) relataram que a mistura de glyphosate com fomesafen resultou em injúrias às plantas de soja. A porcentagem de fitointoxicação variou de $20 \%$ a $28 \%$, quando utilizado o sal de trimetilsulfonio de glyphosate, e de $13 \%$ a $17 \%$ para o de isopropilamina. Como constatado em outros estudos, a fitointoxicação observada foi temporária. Os resultados apresentados por GREY e RAYMER (2002) também revelam que as injúrias visuais nas plantas de soja são variáveis de acordo com o sal de glyphosate utilizado.

Para as plantas de $C$. benghalensis (Tabela 4), aos 3 DAA, a mistura de glyphosate com os herbicidas flumioxazin e lactofen, lactofen e fomesafen isolados, fomesafen mais lactofen e chlorimuron-ethyl mais lactofen, resultaram nas maiores notas de fitointoxicação. No entanto, as plantas recuperaramse da ação desses herbicidas, refletindo em baixas porcentagens de controle nas avaliações seguintes. Para aqueles de ação lenta na planta, como glyphosate, chlorimuron e imazethapyr, de modo geral, mesmo com o decorrer do tempo, os sintomas não se agravaram. Apenas o glyphosate, quando aplicado isolado, ocasionou aumento dos sintomas resultando em maior controle das plantas, embora com valor $(45 \%)$ muito abaixo do necessário para proporcionar um controle aceitável. A aplicação seqüencial desse herbicida não controlou com maior eficácia, proporcionando média inferior à sua aplicação única. Nas plantas tratadas com glyphosate sozinho e em mistura com lactofen verificou-se menor acúmulo de massa, diferindo unicamente do herbicida imazetahpyr, quando aplicado sozinho. 


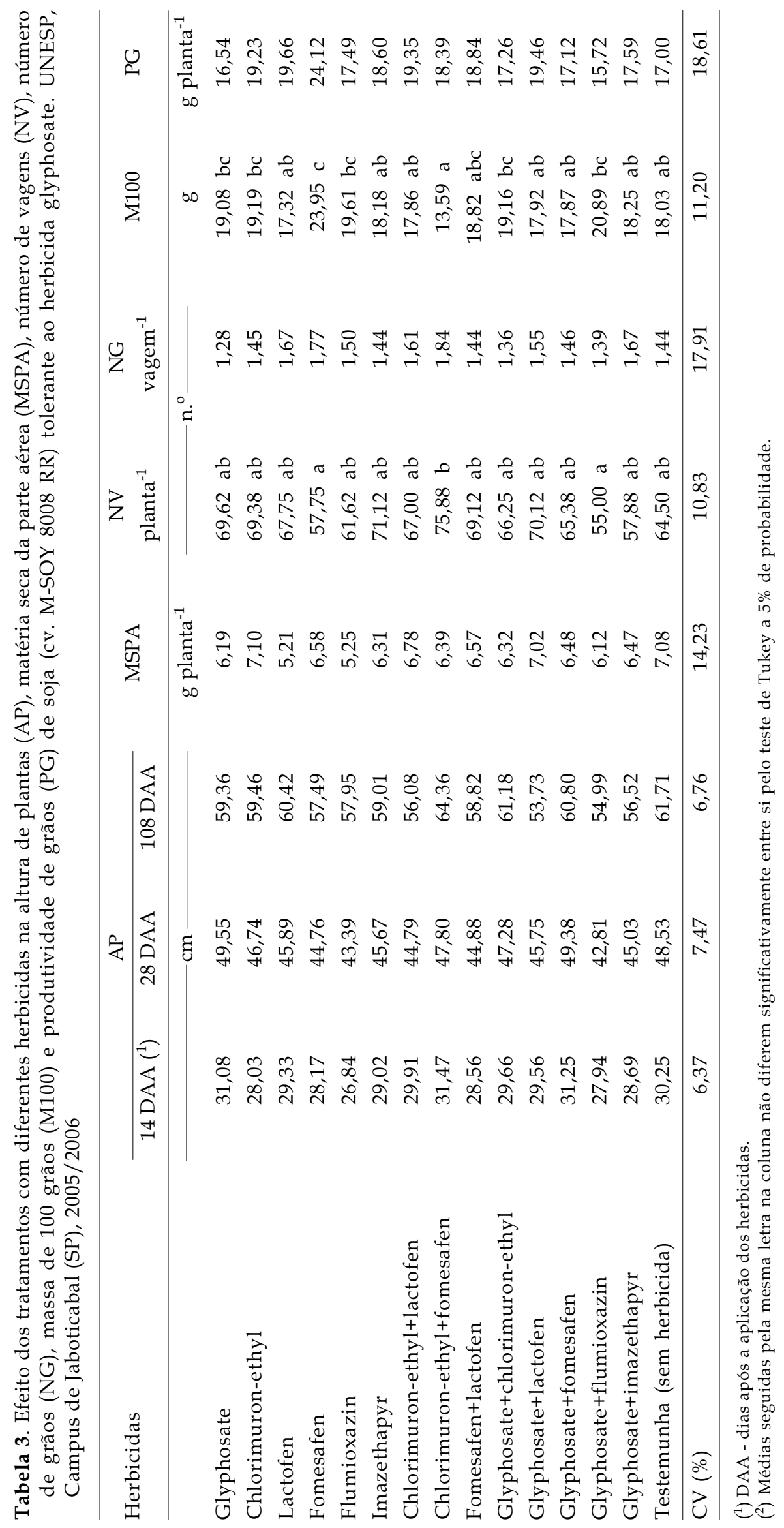




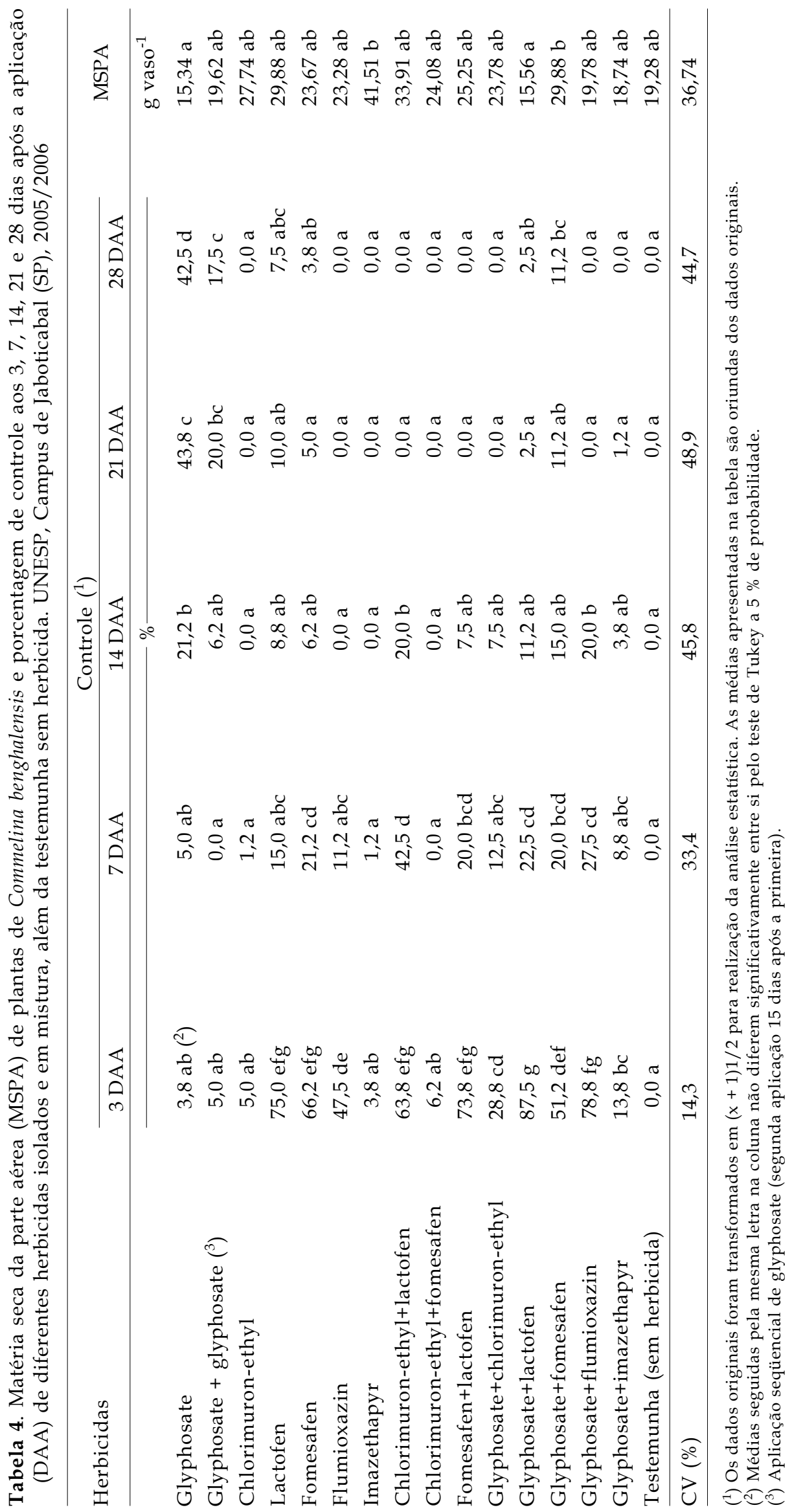


Para o controle eficaz de $C$. benghalensis em soja $R R$, deve-se efetuar a aplicação seqüencial de glyphosate, nas doses de $0,96 \mathrm{~kg} \mathrm{ha}^{-1}$ mais $0,72 \mathrm{~kg} \mathrm{ha}^{-}$ ${ }^{1}$, sendo a primeira aplicação realizada até 20 dias após a emergência das plântulas e a segunda, de 15 a 20 dias após a primeira (Agrofit, 2006). Entretanto, como demonstrado na Tabela 4, C. benghalensis apresentou tolerância ao glyphosate. Devido a essa tolerância natural, será necessário empregar outras técnicas de manejos em áreas de soja transgênica. O estádio de desenvolvimento das plantas no momento da aplicação do herbicida é um fator importante no controle dessa espécie. Nesse estudo, trabalhando-se com plantas, provenientes de sementes e de 4 a 6 folhas totalmente expandidas, não houve controle satisfatório. Talvez, aplicando-se em plantas com menor número de folhas os resultados fossem mais satisfatórios.

ELLIS e GRIFFIN (2003) observaram que a mistura de glyphosate com os herbicidas acifluorfen, oxasulfuron, chlorimuron, fomesafen ou lactofen, em doses reduzidas, aumentou o controle das plantas de Euphorbia heterophylla, S. spinosa, I. lacunosa e S. exaltata. No entanto, isto ocorreu apenas quando as plantas encontravam-se com tamanho acima do recomendado para controle com glyphosate sozinho, comprovando que o estádio de desenvolvimento das plantas daninhas no momento da aplicação influencia na eficácia do produto quando aplicado sozinho e em mistura com outros herbicidas.

Starke e Oliver (1998) verificaram que a adição de fomesafen à calda reduziu o controle de Echinochloa crus-galli $\mathrm{e}$ I. hederacea pelo glyphosate, demonstrando que há antagonismo entre os dois herbicidas. Nesse mesmo trabalho, através da técnica do carbono marcado ( ${ }^{14} \mathrm{C}$-glyphosate), constatou-se que o fomesafen interferiu na absorção e na translocação do glyphosate pelas plantas de E. crus-galli e Abutilon theophrasti, e na absorção pelas de $I$. hederacea. Para a mistura de glyphosate com chlorimuron a interação foi aditiva, para todas as espécies citadas anteriormente.

\section{CONCLUSÕES}

1. Alguns herbicidas e suas misturas causaram injúrias no desenvolvimento inicial da soja.

2. Não houve redução na produtividade da soja devido à aplicação desses produtos.

3. A mistura de glyphosate com os herbicidas chlorimuron-ethyl, lactofen, fomesafen, flumioxazin e imazethapyr, assim como a sua aplicação seqüencial, não foi eficaz no controle de $C$. benghalensis, quando as plantas estavam no estádio de 4 a 6 folhas totalmente expandidas.

\section{REFERÊNCIAS}

AGROFIT. Roundup Ready. Disponível em <http:// www.extranet.agricultura.gov.br/ agrofit_cons/ principal_agrofit_cons>. Acesso em 21 de agosto de 2006.

ATEH, C. A.; HARVEY, R. G. Annual weed control by glyphosate in glyphosate-resistant soybean (Glycine max). Weed Technology, Champaign, v.13, n.2, p.394-398, 1999.

BENNETT, A. C.; SHAW, D. R.; SCHRAER, S. M. Effect of conventional herbicide programs and irrigation on glyphosate-tolerant soybean yield. In: Southern Weed Science Society Annual Meeting, 51., 1998. Proceedings... Birmingham: Southern Weed Science Society, 1998. p.270-271.

ELLIS, J. M.; GRIFFIN, J. L. Glyphosate and broadleaf herbicide mixtures for soybean (Glycine max). Weed Technology, Champaign, v.17, n.1, p.21-27, 2003.

GREY, T. L.; RAYMER, P. Sicklepod (Senna obtusifolia) and red morningglory (Ipomoea cocinea) control in glyphosate-resistant soybean with narrow rows and postemergence herbicide mixtures. Weed Technology, Champaign, v.16, n.3, p.669-674, 2002.

LANIE, A. J.; GRIFFIN, J. L.; VIDRINE, P. L.; REYNOLDS, D. B. Weed control with nonselective herbicides in soybean (Glycine max) stale seedbed culture. Weed Technology, Champaign, v.8, n.1, p.159-164, 1994.

LICH, J. M.; RENNER, K. A.; PENNER, D. Interaction of glyphosate with postemergence soybean (Glycine max) herbicides. Weed Science, Champaign, v.45, n.1, p.12-21, 1997.

LACERDA, A. L. S.; VICTORIA FILHO, R. Curvas dose-resposta em espécies de plantas daninhas com o uso do herbicida glyphosate. Bragantia, Campinas, v.63, n.1, p.73-79, 2004.

MONQUERO, P. A.; CURY, J. C.; CHRISTOFFOLETI, P. J. Controle pelo glyphosate e caracterização geral da superfície foliar de Commelina benghalensis, Ipomoea hederifolia, Richardia brasiliensis e Galinsoga parviflora. Planta Daninha, Viçosa, v.23, n.1, p.123-132, 2005a.

MONQUERO, P. A.; CURY, J. C.; CHRISTOFFOLETI, P. J. Absorção, translocação e metabolismo do glyphosate por plantas tolerantes e suscetíveis a este herbicida. Planta Daninha, Viçosa, v. 22, n.3, p.445-451, 2005b.

NORRIS, J. L.; SHAW, D. S.; SNIPES, C. E. Weed control from herbicide combination with three formulations of glyphosate. Weed Technology, Champaign, v.15, n.3, p.552-558, 2001.

RAYMER, P. L.; GREY, T. L. Challenges in comparing transgenic and nontransgenic soybean cultivars. Crop Science, Madison, v.43, n.5, p.1584-1589, 2003.

STARKE, R. J.; OLIVER, L. R. Interaction of glyphosate with chlorimuron, fomesafen, imazethapyr, and sulfentrazone. Weed Science, Champaign, v.46, n.6, p.652-660, 1998. 
VARGAS, L.; ROMAN, E.S.; RIZZARDI, M.A.; TOLDEO, R.E.B. de. Manejo de azevém resistente ao glyphosate em pomares de maçã com herbicida Select (clethodim). Revista Brasileira de Herbicidas, Passo Fundo, v.5, n.1, p.27-31, 2006.

VIDRINE, P. R.; GRIFFIN, J. L.; BLOUIN, D. C. Evaluation of reduced rates of glyphosate and chlorimuron in glyphosateresistant soybean (Glycine max). Weed Technology, Champaign, v.16, n.4, p.731-736, 2002.

WEBSTER, E. P.; BRYANT, K. J.; EARNEST, LARRY, D. Weed control and economics in nontransgenic and glyphosateresistant soybean (Glycine max). Weed Technology, Champaign, v.13, n.3, p.586-593, 1999. 